\title{
INCIDÊNCIA DE PODRIDÃO-BRANCA EM FRUTOS DE MACIEIRA COM E SEM FERIMENTOS ${ }^{1}$ \\ JANAÍNA PEREIRADOS SANTOS², ADRIANA REGINA CORRENT ${ }^{3}$, ONOFRE BERTON $^{4}$, LÍGIALOSS SCHWARZ ${ }^{5}$, FREDERICO DENARDI ${ }^{6}$
}

RESUMO-Este estudo teve como objetivo avaliar a incidência da podridão-branca (Botryosphaeria dothidea) em frutos de dois genótipos de macieira submetidos à inoculação artificial, na ausência e na presença de ferimentos provocados pela mosca-das-frutas (Anastrepha fraterculus) e por estilete. O experimento foi conduzido no laboratório de Entomologia da Epagri/Estação Experimental de Caçador, na safra 2005/2006. No estudo, foram utilizados frutos da cv. Catarina (grupo 'Fuji') e da seleção M-13/00 (grupo 'Gala'). Os tratamentos foram os seguintes: (1) frutos feridos por mosca-das-frutas; (2) frutos feridos com estilete; (3) frutos sem ferimentos, e (4) frutos sem ferimentos pulverizados com água destilada (testemunha). Os tratamentos 1;2 e 3 foram inoculados com B. dothidea. O delineamento experimental foi inteiramente casualizado, com quatro repetições, de quatro frutos por parcela. Na cv. Catarina, o número de lesões de podridão-branca foi maior em relação à $\mathrm{M}-13 / 00$. Os ferimentos nos frutos favoreceram o estabelecimento e o desenvolvimento de lesões da doença.

Termos para indexação: Botryosphaeria dothidea, Malus domestica, Anastrepha fraterculus, fitossanidade.

\section{INCIDENCE OF WHITE ROT IN APPLE FRUITS WITH AND WITHOUT WOUNDS}

ABSTRACT - The relationship between damages of wounds, fruit fly and the incidence of white rot (Botryosphaeria dothidea), inoculated artificially in two apple genotypes was studied. The experiment was carried out at the laboratory of Entomology at Epagri/ Caçador Experimental Station, during the 2005/2006 season. 'Catarina' (group 'Fuji') and the selection M-13/00 (group 'Gala') were tested with the following treatments: (1) fruit submitted to fruit fly; (2) fruit submitted to wounds by needle; (3) fruit without wounds; (4) fruit without wounds sprayed with distilled water (control). The treatments 1,2 and 3 were inoculated with $B$. dothidea. The experiment was conducted under completely randomized design with four replications of four apple fruits each. The frequency of lesions of white rot was larger on cv. Catarina compared to M-13/00. Fruit wounds stimulate the establishment and the development of white rot lesions.

Index Terms: Botryosphaeria dothidea, Malus domestica, Anastrepha fraterculus, phytossanitary.

\section{INTRODUÇÃO}

A produção mundial de maçãs em 2005 foi de 63,4 milhões de toneladas, com o Brasil ocupando o décimo quarto lugar, com 870 mil toneladas, representando 1,33\% da produção total (FAO, 2006). No Brasil, o Estado de Santa Catarina é o principal produtor, com uma área de 18,4 mil hectares, detendo $60 \%$ da produção nacional (Epagri/Cepa, 2005). Concentrada na região Sul, 95\% da produção brasileira provém das cultivares Gala e Fuji, e de mutações destas (Almeida \& Alves, 2006), todas muito suscetíveis a doenças (Santos \& Wamser, 2006).

Em Santa Catarina, os pomares de macieira estão localizados em regiões com precipitação pluviométrica acima de $1.600 \mathrm{~mm}$ anuais, com temperaturas médias durante a primavera em torno de $16^{\circ} \mathrm{C}$ e no verão de $20^{\circ} \mathrm{C}$. Estas condições climáticas conferem à região um ambiente propício para o desenvolvimento de diversas doenças que, se não forem controladas, comprometem a produção (Bleicher, et al., 1986).

As podridões causadas por fungos estão entre os principais problemas fitossanitários que afetam a macieira. No
Sul do Brasil, a podridão-branca, causada pelo fungo Botryosphaeria dothidea (Moug.) Ces. \& De Not., é uma das principais doenças da cultura. A sua ocorrência é mais severa em anos quentes e chuvosos, resultando em elevado índice de podridão de frutos (Berton et al., 2006). No campo, os sintomas mais evidentes são a murcha das folhas, seca dos galhos e morte dos ramos. Em período de seca, a epiderme atacada pode soltarse do lenho, apresentando o sintoma denominado "cancro-depapel” (Melzer \& Berton, 1986). De acordo com Biggs \& Miller (2003), é difícil avaliar a incidência de podridões a campo, devido à similaridade dos sintomas causados por vários patógenos, como Colletotrichum acutatum, C. gloeosporioides, B. dothidea e B. obtusa. Estes autores comentam que estudos de inoculação do patógeno sob condições controladas, em laboratório ou a campo, possibilitam a identificação correta dos sintomas e a caracterização da relação de patogenicidade do hospedeiro.

$O$ fungo $B$. dothidea não necessita de ferimentos para causar infecção, entretanto, na presença destes, ocorre rápida colonização e desenvolvimento de lesões (Brown \& Hendrix, 1981; Melzer \& Berton, 1986; Berton et al., 2006). Em safras cujo

${ }^{1}$ (Trabalho 005-07). Recebido em 03-01-2007. Aceito para publicação em: 17-01-2008.

${ }^{2}$ Eng. Agr.; M.Sc., EPAGRI/Estação Experimental de Caçador, Abílio Franco, C.P. 591, 89500-000, Caçador-SC. E-mail: janapereira@epagri.sc.gov.br. ${ }^{3}$ Eng. Agr., M.Sc., Doutoranda em Fitotecnia, UfRGS, Av. Bento Gonçalves, 7712, 91501-970, Porto Alegre-RS. E-mail:acorrent@hotmail.com.

${ }^{4}$ Eng. Agr., P.hD. EPAGRI/Estação Experimental de Caçador. E-mail: berton@epagri.sc.gov.br.

${ }^{5}$ Acadêmica de Agronomia, Bolsista de Iniciação Científica., UFRGS. E-mail:ligiaschwarz@hotmail.com.

${ }^{6}$ Eng. Agr., M.Sc. EPAGRI/Estação Experimental de Caçador. E-mail: denardi@epagri.sc.gov.br. 
ataque de insetos é intenso, pode haver aumento na incidência de podridão-branca, uma vez que os danos ocasionados por estes podem favorecer a penetração de fungos.

No Brasil, a mosca sul-americana, Anastrepha fraterculus (Wiedemann, 1830) (Diptera: Tephritidae), é a principal praga da macieira (Ribeiro, 1999; Kovaleski et al., 2000). Este inseto provoca danos diretos aos frutos, ocasionando deformações na epiderme e galerias na polpa (Kovaleski et al., 2000), e, indiretamente, seus ferimentos servem de porta de entrada para fungos e bactérias, tornando-os impróprios para a comercialização e consumo. Neste contexto, este estudo teve por objetivo avaliar a incidência da podridão-branca em frutos de macieira da cv. Catarina e da seleção M-13/00 submetidos à inoculação artificial na ausência e na presença de ferimentos provocados por mosca-das-frutas e por estilete.

\section{MATERIAL E MÉTODOS}

O experimento foi conduzido no laboratório de Entomologia da Epagri/Estação Experimental de Caçador, durante a safra 2005/2006. Foram utilizados frutos maduros da cv. Catarina (grupo 'Fuji') e da seleção M-13/00 (grupo 'Gala'). Para assegurar a integridade dos frutos, realizou-se o ensacamento no campo, durante a primavera, quando estes apresentavam $2 \mathrm{~cm}$ de diâmetro, período em que ainda não havia incidência da moscadas-frutas.

O experimento foi composto dos seguintes tratamentos: (1) frutos feridos por mosca-das-frutas e, em seguida, inoculados com B. dothidea; (2) frutos feridos com estilete e, em seguida, inoculados $\operatorname{com} B$. dothidea; (3) frutos sem ferimentos inoculados com $B$. dothidea; (4) frutos sem ferimentos pulverizados com água destilada (testemunha). O delineamento experimental foi inteiramente casualizado, com quatro repetições e quatro frutos por parcela, totalizando 16 frutos por tratamento, num total de 64 frutos por genótipo de macieira.

Para a realização do tratamento 1 , estabeleceu-se em laboratório uma criação de $A$. fraterculus. Como substrato para o desenvolvimento larval dos insetos, foram utilizados frutos de mamão papaia. Os adultos foram mantidos em gaiolas teladas de criação (malha de 0,2 mm) medindo $50 \mathrm{~cm}$ x $50 \mathrm{~cm}$, em sala climatizada, com condições controladas (temperatura de $25 \pm$ $1{ }^{\circ} \mathrm{C}$, UR de $70 \pm 10 \%$ e fotoperíodo de $12 \mathrm{~h}$ ). Como alimento, aos adultos foi fornecida uma dieta composta por partes iguais de açúcar mascavo, proteína de soja e gérmen de trigo.

O fungo $B$. dothidea foi obtido a partir de maçãs 'Fuji' provenientes de pomares comerciais da região de Caxias do SulRS. Os isolados foram colocados em placas de Petri, contendo meio de cultura batata-dextrose-ágar (BDA), e cultivados em câmara de crescimento a $26^{\circ} \mathrm{C}$ sob luz contínua (lâmpada fluorescente), durante sete dias.

Para a produção de conídios, os isolados de $B$. dothidea foram repicados para discos de papel-filtro esterilizado, sobre o meio de cultura BDA nas placas de Petri, permanecendo por 15 dias, a $26^{\circ} \mathrm{C}$, sob luz fluorescente constante. Após este período, os papéis-filtro foram retirados das placas e triturados em almofariz, juntamente com $20 \mathrm{~mL}$ de meio de cultura batata- dextrose (BD), estéril a 5\%. A suspensão obtida foi filtrada através de três camadas de gaze para a remoção dos resíduos de micélio do patógeno. A concentração de conídios na suspensão foi determinada com um hemocitômetro e ajustada para $1 \times 10^{6}$ conídios. $\mathrm{mL}^{-1}$ para utilização na inoculação dos frutos.

Depois de colhidos no ponto ideal de consumo (nível de degradação de amido entre 5 e 6), os frutos foram armazenados em câmara fria $\left(1^{\circ} \mathrm{C} \pm 1^{\circ} \mathrm{C}\right)$ até o início das inoculações. Vinte e quatro horas antes das inoculações, os frutos foram retirados da câmara fria e desinfestados com hipoclorito de sódio a $0,5 \%$, lavados com água destilada e colocados para secar em papel toalha. Em seguida, os frutos do tratamento 1 foram colocados em gaiolas entomológicas e expostos ao ataque da mosca-dasfrutas, que, por sua vez, faz ferimentos na epiderme dos frutos através das puncturas para oviposição. Cada genótipo foi infestado separadamente pela mosca-das-frutas. Em cada gaiola havia 30 fêmeas com aproximadamente 25 dias, conforme metodologia de Branco (1998). Os frutos permaneceram 48 horas nas gaiolas a $25^{\circ} \mathrm{C}$ e fotoperíodo de 12 horas. Em seguida, foram retirados e, novamente desinfestados com hipoclorito de sódio a $0,5 \%$ e lavados com água destilada. Nos frutos do tratamento 2, a inoculação foi feita via ferimentos feitos com auxílio de estilete, conforme metodologia de Camilo (1989) e Denardi et al. (2003). Utilizou-se um estilete com espessura similar ao ovipositor da mosca-das-frutas. Após esse processo, imediatamente, os frutos dos tratamentos 1; 2 e 3 foram inoculados por pulverização com $25 \mu \mathrm{L}$ de uma suspensão de esporos de $B$. dothidea, contento $1.10^{6}$ conídios. $\mathrm{mL}^{-1}$. Os frutos do tratamento 4 foram pulverizados apenas com água destilada.

Após a inoculação, os frutos de cada repetição dos tratamentos foram armazenados em sacos de plástico previamente umedecidos e transferidos para incubadora a $25^{\circ} \mathrm{C}$ e fotoperíodo de 12 horas. A cada sete dias, os frutos foram submetidos à avaliação do número de lesões de podridão branca, num total de três avaliações. Os totais de lesões por genótipo e por tipo de injúria nos frutos foram submetidos ao teste de Qui-quadrado para avaliar as possíveis associações entre as variáveis. $\mathrm{O}$ tratamento 4 não foi submetido à análise por não apresentar lesões de podridão-branca em nenhum dos genótipos avaliados.

\section{RESULTADOS E DISCUSSÃO}

Em relação às épocas de avaliação, tanto na 'Catarina' quanto na M-13/00, o fungo desenvolveu-se mais rápido em frutos submetidos a ferimentos por mosca-das-frutas e por estilete. Em ambos os tratamentos, as primeiras lesões de podridão-branca apareceram 14 dias após a inoculação. Apesar de o patógeno também desenvolver-se em frutos sem ferimentos, as primeiras lesões de podridão foram observadas posteriormente, apenas na última avaliação, aos 21 dias após a inoculação.

$\mathrm{Na}$ 'Catarina', houve associação positiva entre a freqüência de lesões de podridão-branca e o dano causado por mosca-das-frutas (Tabela 1). Nesta cultivar, o número de lesões de podridão-branca nos frutos submetidos à mosca-das-frutas foi muito superior ao número de lesões desenvolvidas em frutos feridos com estilete e nos frutos sem ferimentos. Fundamentando- 
se nessa observação, o controle de agentes bióticos ou abióticos que possam promover ferimentos nos frutos, poderá reduzir a incidência de podridão-branca em maçãs.

Denardi et al. (2003) e Berton et al. (2005) estudaram o progresso da podridão-amarga (Glomerella cingulata (Stoneman) Spaulding \& Schrenk) em frutos de diferentes genótipos de macieira, com e sem ferimentos, e verificaram que a doença se estabeleceu mais rapidamente em frutos com ferimentos, indicando que a baixa incidência de lesões na ausência destes é um bom indicativo da importância de se manterem os frutos protegidos do ataque de insetos, principalmente da moscadas-frutas.

Em relação aos frutos de M-13/00, houve uma associação positiva entre a freqüência de lesões de podridão-branca e o dano causado por estilete. Os frutos desta seleção apresentaram mais lesões de podridão-branca quando feridos com estilete (Tabela 1). De acordo com Melzer \& Berton (1986), o grau de ataque de $B$. dothidea varia consideravelmente com a cultivar. Estes autores, estudando a suscetibilidade de várias cultivares de macieira a $B$. dothidea, verificaram que a cv. Royal Red Delicious (clone mutante da 'Delicious' e parental masculino da 'Fuji') foi a mais suscetível, e as cultivares Gala e Rainha, ambas parentais da 'Golden Delicious', foram as mais resistentes.

TABELA 1 - Número de lesões de podridão-branca em frutos de 'Catarina' e de M-13/00, submetidos a três diferentes tratamentos.

\begin{tabular}{lcccc}
\hline \multirow{2}{*}{ Genótipo } & Mosca-das-frutas & Estilete & Sem ferimentos & Total \\
\hline Catarina & 43,76 & 20,93 & 21,35 & 86,04 \\
M-13/00 & 1,06 & 11,84 & 1,06 & 13,96 \\
\hline Total & 44,82 & 32,77 & 22,41 & \\
\hline
\end{tabular}

As freqüências de lesões nos genótipos e formas de injúria avaliadas diferem significativamente $(\mathrm{P}<0,01)$ das esperadas ao acaso, pelo teste Quiquadrado $(94,75)$, com 2 graus de liberdade.

\section{CONCLUSÕES}

1-Os ferimentos nos frutos favorecem o estabelecimento e o desenvolvimento de lesões da podridão-branca.

2-A cultivar Catarina (grupo 'Fuji') é mais suscetível à podridão-branca do que a seleção M-13/00 (grupo 'Gala').

\section{AGRADECIMENTO}

Os autores agradecem ao Eng. Agr. M.Sc. Cristiano Nunes Nesi, pelo auxílio nas análises estatísticas.

\section{REFERÊNCIAS}

ALMEIDA, G.V.B. de; ALVES, A.A. Mercado de maçã: situação atual, ameaças, oportunidades e estratégias para o futuro. In: SEMINÁRIONACIONAL SOBRE FRUTICULTURADE CLIMA TEMPERADO, 7., 2006, São Joaquim. Resumos... São Joaquim: Epagri, 2006. p.56-65.
BERTON, O.; BLEICHER, J.; BONETI, T.J. da S.; KATSURAYAMA, Y. Doenças fúngicas dos ramos: podridãobranca - Botryosphaeria dothidea (Moug.) Ces. \& De Not. (=B. ribis Gross. \& Dug.) (=B. berengeriana De Not.), (Dothiorella sp. Sacc). In: EPAGRI. A cultura da macieira. Florianópolis: Epagri, 2006. p.567-571.

BERTON, O.; SANTOS, J. P.; DENARDI, F. Relação entre danos de mosca-das-frutas e a incidência de podridão-amarga em frutos de macieira. In: ENCONTRO NACIONAL DE FRUTICULTURA DE CLIMA TEMPERADO, 8., 2005, Fraiburgo. Anais... Caçador: Epagri, 2005.v.2, p.73-73.

BIGGS, A.R.; MILLER, S.S. Relative susceptibility of selected apple cultivars to Botryosphaeria dothidea. HortScience, Alexandria, v.38, n.3, p.400-403, 2003.

BLEICHER, J.; MELZER, R.; BERTON, O.; BONETI, J.I.S; DRIESSEN, A.C. Doenças da macieira. In: EPAGRI. Manual da cultura da macieira. Florianópolis: Empasc, 1986. p.380-442.

BRANCO, E. da S. Resistência de genótipos de macieira à moscadas-frutas Anastrepha fraterculus (Wied., 1830) (Diptera: Tephritidae). 1998. 119f. Dissertação (Mestrado em Ciências) Escola Superior de Agricultura Luiz de Queiroz, Universidade de São Paulo, Piracicaba, 1998.

BROWN, E.A.; HENDRIX, F.F. Pathogenecity and histopathology of Botryosphaeria dothidea on apple stems. Phytopathology, St. Paul, v.71, p.375-379, 1981.

CAMILO, A.P. Genetic resistance in Malus to Glomerella cingulata (Stoneman) Spaulding \& Von Schrenk: sources of resistance, leaf infection, progeny evaluation, and pathogenecity. 1989. 116f. Tese (Doutorado) - Cornell University, Ithaca, 1989.

DENARDI, F.; BERTON, O.; SPENGLER, M.M. Resistência genética à podridão amarga em maçãs, determinadas pela taxa de desenvolvimento da doença em frutos com e sem ferimentos. Revista Brasileira de Fruticultura, Jaboticabal, v.25, n.3, p.375$558,2003$.

EPAGRI/CEPA. Síntese anual da agricultura de Santa Catarina 2004-2005. Disponível em: <http://cepa.epagri.sc.gov.br/ Publicacoes/sintese_2005/maca_2005.pdf $>$. Acesso em: 13 nov. 2006.

FAO. Statistical databases. Disponível em: $<$ http://www.fao.org/ waicent/portal/statistics_em.asp>.Acesso em: 20 jul. 2006.

KOVALESKI, A.; SUGAYAMA, R.L.; MALAVASI, A. Controle químico em macieiras. In: MALAVASI, A.; ZUCCHI, R.A. (Eds.). Mosca-das-frutas de importância econômica no Brasil: conhecimento básico e aplicado. Ribeirão Preto: Holos, 2000. p.135-141. 
MELZER, R.R.; BERTON, O. Incidência de Botryosphaeria berengeria na cultura da macieira (Malus domestica) no estado de Santa Catarina, Brasil. Fitopatologia Brasileira, Brasília, v.11, n.4, p.891-898, 1986.

RIBEIRO, L.G. Principais pragas da macieira: mosca-das-frutas (Anastrepha fraterculus). In: BONETI, J.I. da S.; RIBEIRO, L.G.; KATSURAYAMA, Y. Manual de identificação de doenças e pragas da macieira. Florianópolis: Epagri, 1999. p.97-102.
SANTOS, J.P.; WAMSER, A.F. Efeito do ensacamento de frutos sobre danos causados por fatores bióticos e abióticos em pomar orgânico de macieira. Revista Brasileira de Fruticultura, Jaboticabal, v.28, n.2, p.168-171, 2006.

SUTTON, T.B.; ARAUZ, L.F. Influence of temperature and moisture on germination of ascospores and conidia of Botryosphaeria dothidea. Plant Disease, St. Paul, v.75, p.11461149, 1991. 